\title{
Proliferation and differentiation of neural stem cells on lysine-alanine sequential polymer substrates
}

\author{
Jyh-Horng Wang ${ }^{\mathrm{a}}$, Chih-Huang Hung ${ }^{\mathrm{b}}$, Tai-Horng Young ${ }^{\mathrm{b}, *}$ \\ ${ }^{a}$ Department of Orthopedic surgery, National Taiwan University Hospital, Taipei 100, Taiwan \\ ${ }^{\mathrm{b}}$ Institute of Biomedical Engineering, College of Medicine and College of Engineering, National Taiwan University, Taipei 100, Taiwan
}

Received 15 October 2005; accepted 2 February 2006

Available online 3 March 2006

\begin{abstract}
The purpose of this study was to explore the phenotypic potential of embryonic rat cerebral cortical stem cells by inducing differentiation on lysine-alanine sequential (LAS) polymer substrates at neurosphere level. LAS polymer is a heterologous polymer of lysine and alanine and has been demonstrated to enhance axon growth of neurons in a serum-free medium in vitro. It was found that very few cells migrated outside of the neurospheres but extremely long processes extended from differentiated cells could form a network between remote neurospheres when cells were cultured on LAS substrates at a low density of 120 neurospheres $/ \mathrm{cm}^{2}$ in the serum-free medium. On the contrary, when the neurosphere density was increased to 360 neurospheres $/ \mathrm{cm}^{2}$, many neurosphere-forming cells migrated out from their original aggregate and exhibited short processes morphology. Furthermore, when serum was added to the culture system, the neurosphere-forming cells could be induced into an extensive cellular substratum of protoplasmic cells upon which process-bearing cells spread. Clearly, neurospheres could exhibit different behaviors on LAS substrates according to the complex environmental conditions. Here, we proposed that neurospheres would change their social communication and adopt different strategies to communicate with other neurospheres when they detected each other's presence. Therefore, the mediation of cell behavior on LAS substrates by communication between neurospheres should be taken into account.
\end{abstract}

(C) 2006 Elsevier Ltd. All rights reserved.

Keywords: Lysine-alanine sequential (LAS) polymer; Neural stem cells

\section{Introduction}

The discovery of neural stem cells in the central nervous system (CNS) and their capacity to regenerate functional neural cells have raised hopes for once untreatable diseases and injuries to the brain and spinal cord. In vitro, these neural stem cells or neural progenitor cells can be maintained and expanded in the presence of mitogenic growth factors, and also they have the potential to differentiation to neurons, astrocytes, and oligodendrocytes in response to microenvironmental cues [1-4]. Previous studies suggested that the proliferation and differentiation of embryonic cortical neural stem cells were determined by the effects of extrinsic and intrinsic signals

\footnotetext{
${ }^{*}$ Corresponding author. Tel.: +886223123456x1455; fax: +886223940049

E-mail address: thyoung@ha.mc.ntu.edu.tw (T.-H. Young).
}

coming from substrates, medium components, and several complex interactions among cells [5,6]. For example, epidermal growth factor, basic fibroblast growth factor (bFGF) and hepatocytes growth factor have been shown to induce the proliferation of embryonic precursors of the CNS, leading to the formation of cell clusters, termed neurospheres [2,3,7]. In addition, ciliary neurotrophic factor and insulin-like growth factor-1 have also been reported to function as key cues in regulation of development of neural stem cells $[8,9]$. Besides the diffusible factors, signal coming from cell-cell contact also has an effect on the transition of multipotential neural stem cells to postmitotic cells of different types $[6,10]$. However, little is known what determines the choice of the differentiation pathway taken by neural stem cells on different substrates [10]. It is possible that neural stem cells respond to different substrates, and their fate determination depends on the chemical properties of the substrates. 
Numerous natural and synthetic polymers have been used as substrates or scaffolds for peripheral and central nerve regeneration in vitro or in vivo [11,12]. For example, poly-D-lysine (PDL) is the most common polymer substrate used for culture of CNS neurons because of its efficacy and economy [13-17]. Compared to PDL, lysine-alanine sequential (LAS) polymer is a heterologous polymer of lysine and alanine. Brewer et al. reported that LAS enhanced better axon growth of neurons in a serum-free medium than PDL did [18]. Therefore, this study examined whether the differentiation behavior of neural stem cells responded differently to LAS and PDL substrates under serum-free conditions. Neural stem cells, isolated from embryonic rat cerebral cortices, were cultured on LAS and PDL substrates at the neurosphere level. Furthermore, the effects of neurosphere density and serum proteins on the differentiation of neural stem cells on LAS substrates were investigated. Finally, we proposed the mediation of cell behavior on LAS substrates by communication between neurospheres. The information should be useful for the development of biomaterials to regulate the proliferation and differentiation of neural stem cells and their eventual manipulation to replace lost or dysfunctional neurons following trauma or disease.

\section{Materials and methods}

\subsection{Preparation of $L A S$ and $P D L$ substrates}

LAS polymer was kindly supported by Professor Brewer [18] and its synthesis was described elsewhere [20]. The LAS polymer was dissolved at $1 \mathrm{mg} / \mathrm{ml}$ in distilled water and preserved at $-20^{\circ} \mathrm{C}$ as the stock solution. After thawing, the stock solution was diluted to $50 \mu \mathrm{g} / \mathrm{ml}$. PDL was purchased from Sigma and diluted to $50 \mathrm{ng} / \mathrm{ml}$ in phosphate-buffered saline (PBS). Before cell culture, 24-well tissue culture polystyrene (TCPS) plates (Corning, New York, USA) were covered with $1 \mathrm{ml}$ of LAS or PDL diluted solution and incubated for $12 \mathrm{~h}$, after which time excess solution was removed by suction and dried for another hour.

\subsection{Isolation and culture of cortical neural stem cells}

Cortical neural stem cells were prepared from pregnant Wistar rat embryos on days 14-15 according to a protocol detailed previously $[5,6]$. Briefly, rat embryonic cerebral cortices were dissected, cut into small pieces and mechanically triturated in cold Hank's balanced salt solution containing $5.4 \mathrm{mM} \mathrm{KCl}, 0.3 \mathrm{~mm} \quad \mathrm{Na}_{2} \mathrm{HPO}_{4} \cdot 7 \mathrm{H}_{2} \mathrm{O}, 0.4 \mathrm{mM} \quad \mathrm{KH}_{2} \mathrm{PO}_{4}$, $4.2 \mathrm{~mm} \mathrm{NaHCO}_{3}, 0.5 \mathrm{~mm} \mathrm{MgCl}_{2} \cdot 6 \mathrm{H}_{2} \mathrm{O}, 0.6 \mathrm{~mm} \mathrm{MgSO}_{4} \cdot 7 \mathrm{H}_{2} \mathrm{O}, 137 \mathrm{~mm}$ $\mathrm{NaCl}$, and $5.6 \mathrm{~mm}$ D-glucose. The dissociated cells were collected by centrifugation and were resuspended in a serum-free medium containing DMEM-F12, $8 \mathrm{~mm}$ glucose, glutamine, $20 \mathrm{~mm}$ sodium bicarbonate, $15 \mathrm{~mm}$ HEPES and $\mathrm{N}_{2}$ supplement $(25 \mu \mathrm{g} / \mathrm{ml}$ insulin, $100 \mu \mathrm{g} / \mathrm{ml}$ human apotransferrin, $20 \mathrm{~nm}$ progesterone, $30 \mathrm{~nm}$ sodium selenite, $\mathrm{pH}$ 7.2) [19]. The number of live cells was counted by trypan blue exclusion assay in a hemocytometer.

Cerebral cortical neural stem cells were purified and cultured in T25 culture flasks (Corning, NY, USA) at a density of $50,000 \mathrm{cells} / \mathrm{cm}^{2}$ in the above culture medium in the presence of bFGF at a concentration of $20 \mathrm{ng} / \mathrm{ml}$. Cultures were maintained at $37^{\circ} \mathrm{C}$ in a humidified atmosphere of $95 \%$ air $/ 5 \% \mathrm{CO} 2$. After $1-3 \mathrm{~d}$ in vitro, cells were undergoing cell division and the proliferating cells formed the neurospheres, which were suspended in the medium. Subsequently, adherent cells were discarded and suspended neurospheres were collected by centrifugation, mechanically dissociated and subcultured as single cells in a new T25 culture flask at a density of $50,000 \mathrm{cells} / \mathrm{cm}^{2}$ in the fresh culture medium containing the same concentration of bFGF. These cells grew into new spheres in the subsequent $2-3 \mathrm{~d}$, that is, single cells proliferated and formed new neurospheres. The procedure of subculture was repeated again to achieve the purified cortical neural stem cells and proliferating neurospheres. The plasticity of these purified cortical neural stem cells was identified by the method of immunocytochemistry with anti-nestin, anti-NSE, and antiGFAP, which has been reported in a previous publication [10].

Subsequently, neurospheres were seeded on LAS- or PDL-coated wells at 120 neurospheres $/ \mathrm{cm}^{2}$ in the serum-free medium after bFGF withdrawal for investigating the effects of substrates on neural stem cells. In addition, the effects of higher neurosphere density (at 360 neurospheres/ $\mathrm{cm}^{2}$ under serum-free conditions) and serum proteins (10\% fetal bovine serum (FBS) at 120 neurospheres $/ \mathrm{cm}^{2}$ ) on the differentiation of neural stem cells on LAS substrates were included in this study. The empty TCPS wells without coating were used as controls. At indicated time points, morphologies of cultured neurospheres were observed under a phase contrast microscope (Zeiss LAMBDA 10-2, Germany).

\subsection{Quantification of process growth}

Digital photomicrographs were taken of random fields of neurospheres at indicated experimental points. Quantification of process growth was evaluated by the end-to-end distance of extensive processes. The lengths of $10-15$ longest processes (neurites from neurons or processes from glial cells) per neurosphere were estimated from the edge of the neurospheres to the tip of the processes in linear form. Process lengths were measured by tracing the processes using NIH Image software (ImageJ). The length of processes of 20 independent neurospheres was calculated at each experiment, and the means and standard error of mean (SEM) were also calculated $[21,22]$.

\subsection{MTT assay}

The cell viability was determined by the MTT (3-(4,5-dimethylthiazol2-yl)-diphenyl tetrazolium bromide; Sigma) colorimetric assay $[23,24]$. Briefly, the culture medium was removed at the indicated time points, and the cells were incubated with $0.1 \mathrm{ml}$ of MTT solution $(2 \mathrm{mg} / \mathrm{ml}$ in PBS) for $3 \mathrm{~h}$ at $37^{\circ} \mathrm{C}$. After incubation, the MTT solution was aspirated and the formazan reaction products were dissolved in dimethyl sulfoxide and shaken for $20 \mathrm{~min}$. The optical density of the formazan solution was read on an ELISA plate reader (ELx 800, BIO-TEK) at $570 \mathrm{~nm}$. MTT conversion data were expressed as fold growth relative to seeding neurospheres.

\subsection{Immunocytochemistry}

For immunocytochemical characterization, cultured cells were fixed in ice-cold 4\% paraformaldehyde in PBS for $20 \mathrm{~min}$ and washed three times in PBS at indicated time points. After fixing, cells were incubated with primary antibodies diluted in PBS containing $0.3 \%$ triton-X-100 and $10 \%$ bovine serum albumin for $2 \mathrm{~h}$ at $37^{\circ} \mathrm{C}$. The primary antibodies and their dilution used in this study were mouse anti-nestin monoclonal antibody (anti-nestin; 1:1000; Chemicon, Temecula, CA), mouse anti-neuron specific enolase monoclonal antibody (anti-NSE; 1:250; Chemicon, Temecula, CA), mouse anti- $\beta$-tubulin-III monoclonal antibody (anti$\beta$-tubulin-III; 1:200; Chemicon, Temecula, CA), rabbit anti-microtubuleassociated protein 2 polyclonal antibody (anti-MAP2; 1:500; Chemicon, Temecula, CA), rabbit anti-glial fibrillary acidic protein polyclonal antibody (anti-GFAP; 1:500; Chemicon, Temecula, CA), and mouse anti-oligodendrocyte marker $\mathrm{O} 4$ monoclonal antibody (anti-O4; 1:250; Chemicon, Temecula, CA) [25-28]. FITC- and Rhodamine-conjugated secondary antibodies were used to visualize the signal by reacting with cells for $30 \mathrm{~min}$ at room temperature. The secondary antibodies and their dilution were FITC-conjugated goat anti-mouse IgG (preabsorbed with 
rabbit and rat serum protein; 1:100; Chemicon, Temecula, CA), FITCconjugated donkey anti-rabbit IgG (preabsorbed with rabbit and rat serum protein; 1:100; Chemicon, Temecula, CA) and rhodamineconjugated goat anti-mouse IgG (preabsorbed with rabbit and rat serum protein; 1:100; Chemicon, Temecula, CA). Immunostained cells were visualized by indirect fluorescence under the fluorescent microscope (Axiovert 100TV, Germany).

\section{Results}

\subsection{Behavior of neurospheres on LAS substrates at low density under serum-free conditions}

Fig. 1 shows photomicrographs of neurospheres cultured on LAS substrates at an initial density of 120 neurospheres $/ \mathrm{cm}^{2}$ in the serum-free medium after bFGF withdrawal to allow cell attachment and differentiation. It can be seen that as early as $1 \mathrm{~d}$ in culture, neurospheres attached onto LAS substrates and the differentiating processes extended out from the spheres. Actually, the number and length of processes from differentiated cells within the neurospheres extended their length and territory steadily over the course of $4 \mathrm{~d}$ on LAS substrates. Although neurospheres were discretely distributed on LAS substrates due to the low seeding density, differentiated cell bodies sent out very long, straight, and slender processes into the surrounding areas. Therefore, processes extended from individual neurosphere could form a network between neurospheres after $4 \mathrm{~d}$ of culture. At this time, very few migrating cells were found around the neurospheres. The differentiated neuronal phenotype of these neurospheres was confirmed by immunocytochemical staining, which will be described subsequently.

LAS is a sequential copolymer of lysine and alanine. For comparison, PDL, a homopolymer of lysine molecule and is the most common substrate for neuronal cultures, was used to culture neurospheres under the same conditions. Dissimilar to LAS polymer, PDL could induce more neurosphere-forming cells migrating far away from neurospheres; see Fig. 2. In addition, although these neurospheres were also attached onto PDL substrates after $1 \mathrm{~d}$ of culture and the processes were also becoming abundant and longer to extend to adjacent neurospheres for longer culture times, the processes emanating from neurospheres were not so straight as those on LAS substrates. The differentiated processes exhibited a characteristic of zigzag form.
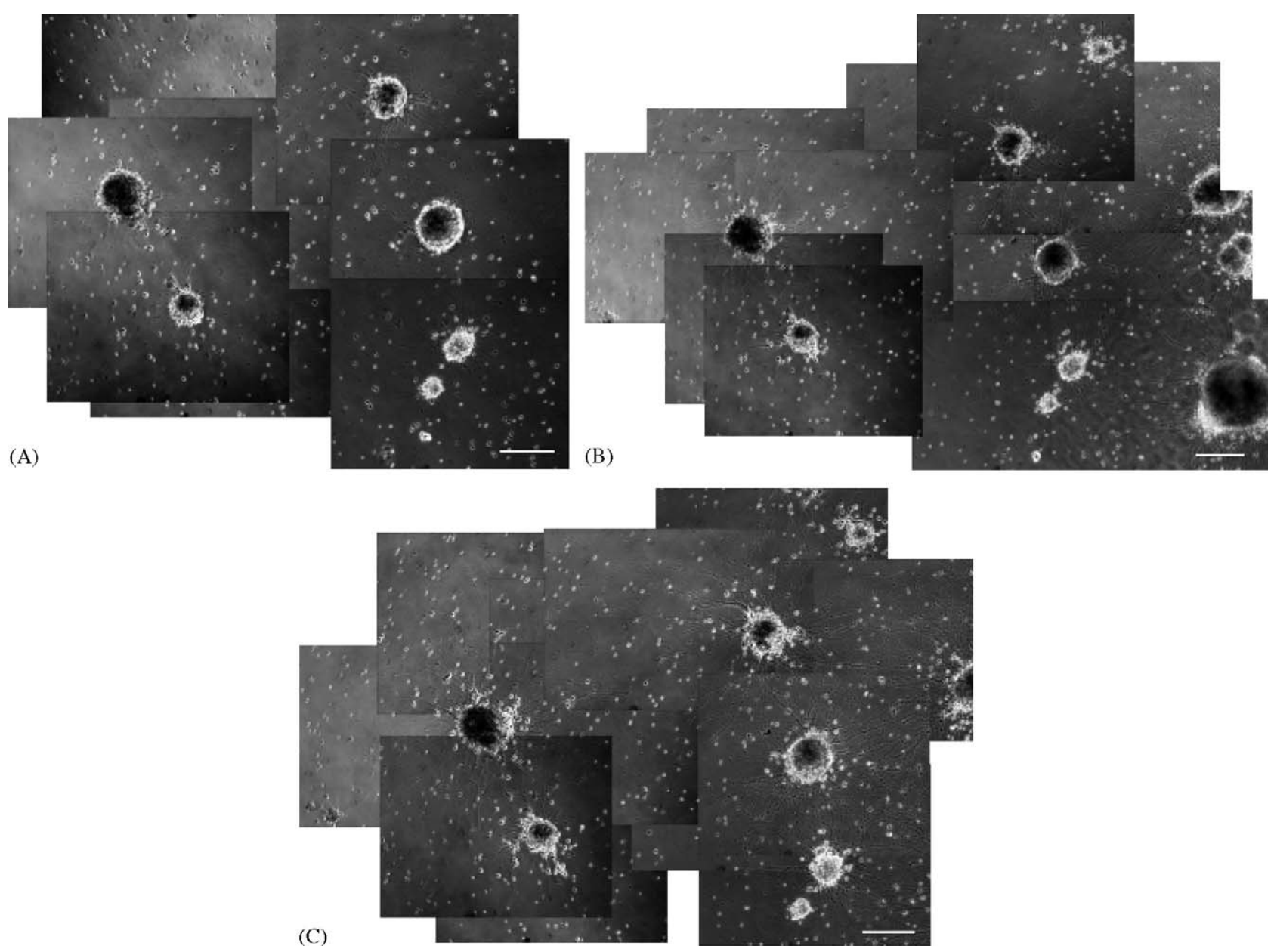

Fig. 1. Photomicrographs illustrate morphology and process growth of embryonic cerebral cortical neurospheres on LAS substrates under serum-free conditions at low neurosphere density $\left(120\right.$ neurospheres $\left./ \mathrm{cm}^{2}\right)$ after culture of (A) $1 \mathrm{~d}$, (B) $2 \mathrm{~d}$, and (C) $4 \mathrm{~d}$. Scale bar $=200 \mu \mathrm{m}$. 

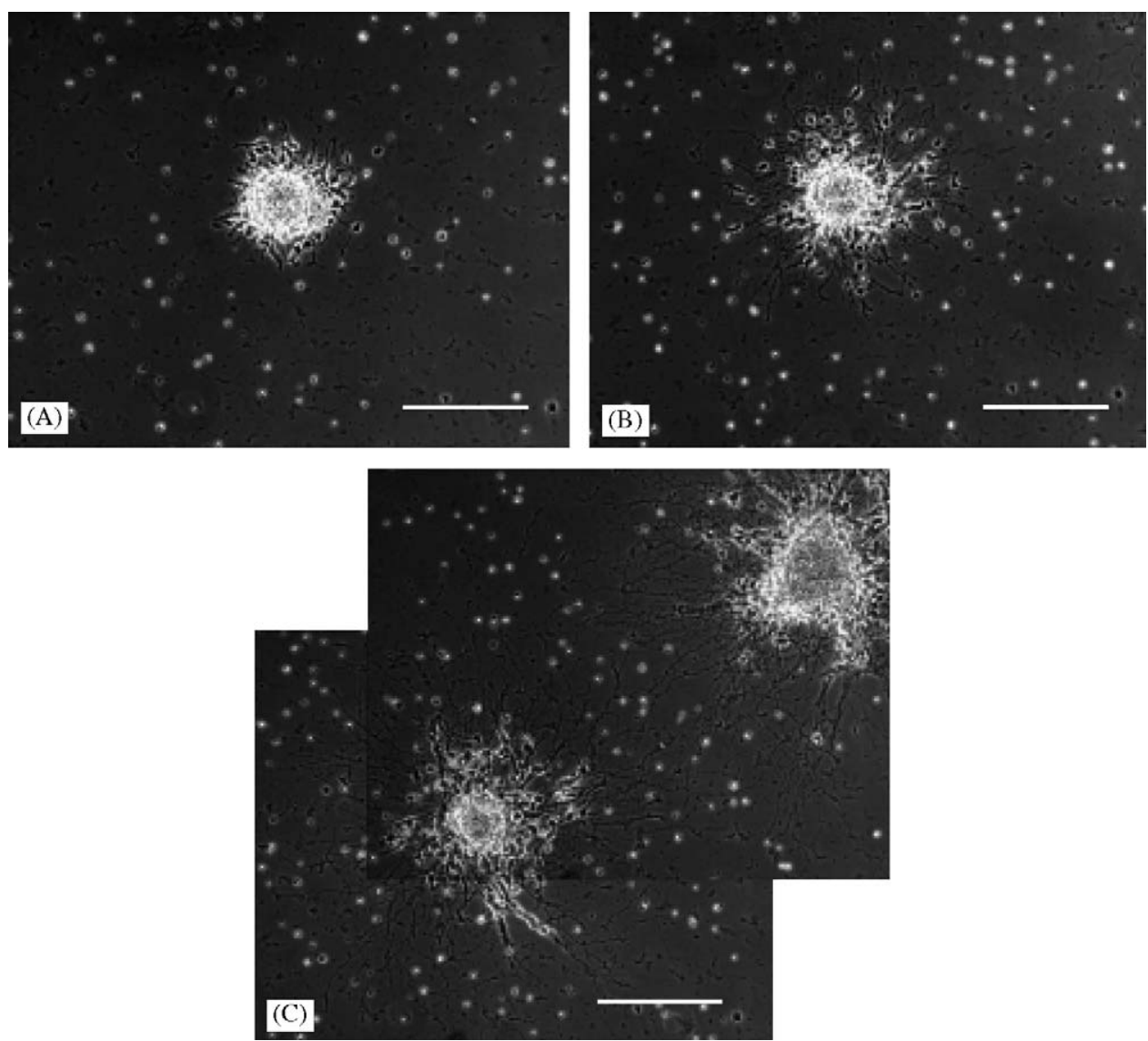

Fig. 2. Photomicrographs illustrate morphology and process growth of embryonic cerebral cortical neurospheres on PDL substrates under serum-free conditions at low neurosphere density (120 neurospheres $\left./ \mathrm{cm}^{2}\right)$ after culture of (A) $1 \mathrm{~d}$, (B) $2 \mathrm{~d}$, and (C) $4 \mathrm{~d}$. Scale bar $=200 \mu \mathrm{m}$.

Fig. 3 shows quantitative analysis of process growth of differentiated cells generated from cerebral cortical neurospheres on LAS and PDL substrates after 1, 2, and $4 \mathrm{~d}$ of culture under serum-free conditions. The lengths of 10-15 longest processes per neurosphere were measured from the edge of the neurospheres to the tip of these processes, i.e., the end-to-end distance. The average process length of 20 independent neurospheres on PDL substrates were $51.2 \pm 1.2,137.9 \pm 2.6$, and $309.6 \pm 6.3 \mu \mathrm{m}$ at 1,2 , and $4 \mathrm{~d}$, respectively. The average process lengths on LAS substrates were $211.6 \pm 4.0,359.9 \pm 5.8$, and $448.8 \pm 6.8 \mu \mathrm{m}$ at 1,2 , and $4 \mathrm{~d}$, respectively, which were significantly longer than those on PDL substrates at each indicated time point $(p<0.001)$.

Because these cultures represent a heterogeneous population of cells at different stages of neuronal and glial development, immunocytochemical staining was performed to analyze differentiated phenotypes after neurospheres were cultured on LAS and PDL substrates for $3 \mathrm{~d}$. Fig. 4 shows that the extensive processes' outgrowth from the neurospheres were both identified as either neuronal filaments (anti- $\beta$-tubulin-III and anti-MAP-2 immunoreactive) or glial processes (anti-GFAP immunoreactive) on LAS and PDL substrates. The monoclonal antibody

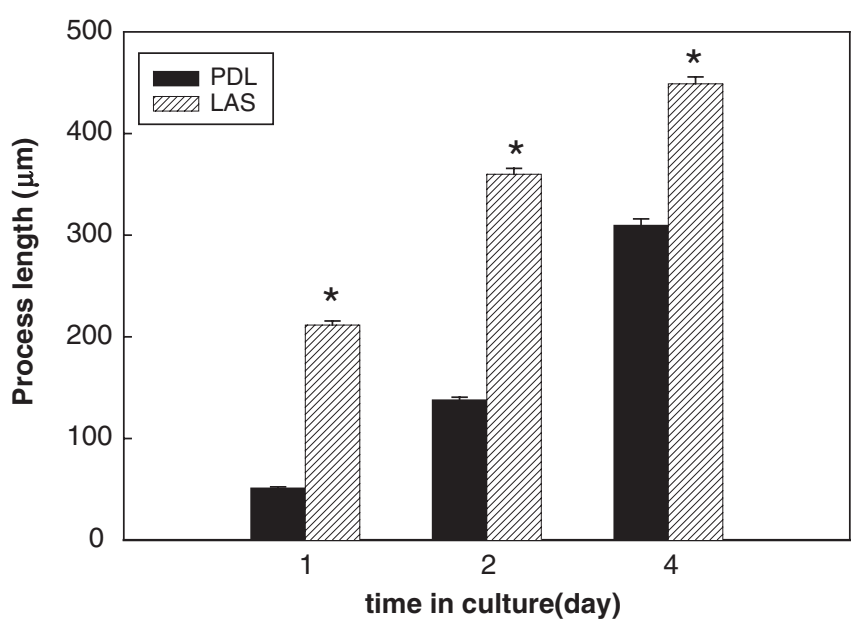

Fig. 3. Quantification of process growth of neurospheres on LAS and PDL substrates under serum-free conditions at 120 neurospheres $/ \mathrm{cm}^{2}$ after 1,2 , and $4 \mathrm{~d}$ of culture. The lengths of $10-15$ longest processes per neurosphere were estimated from the edge of the neurospheres to the tip of the processes in linear form. Values represent means \pm SEM of 20 independent neurospheres. "Significant differences of the average length of processes on LAS substrates compared to that on PDL substrates $(p<0.001)$ as determined by Student's $t$-test. 


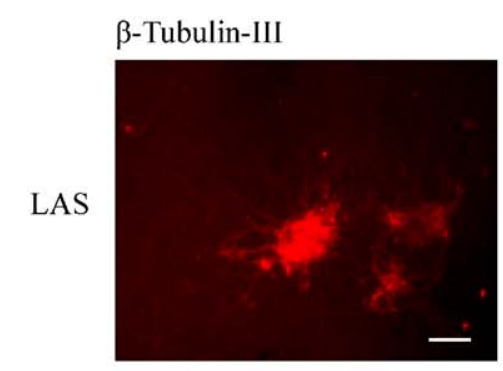

MAP2
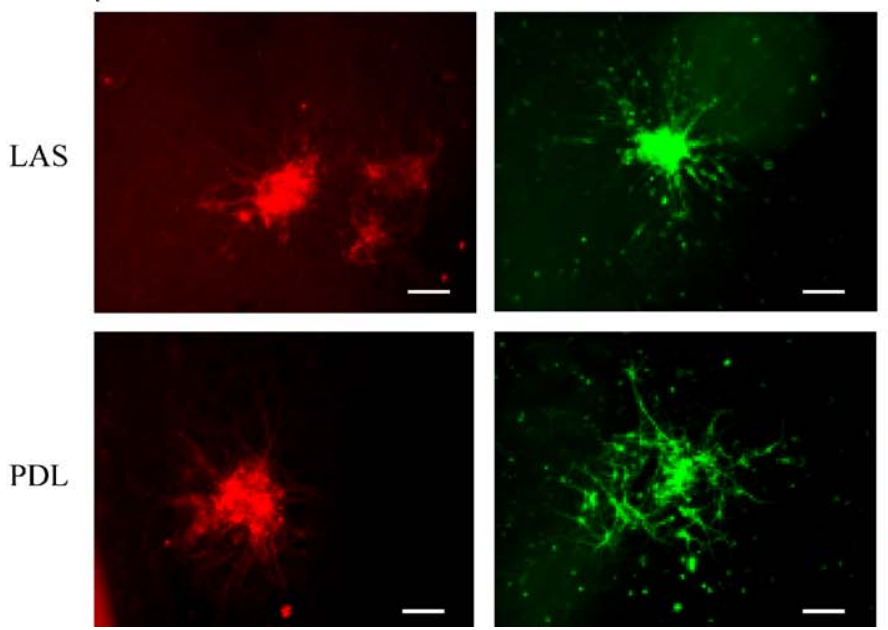

GFAP
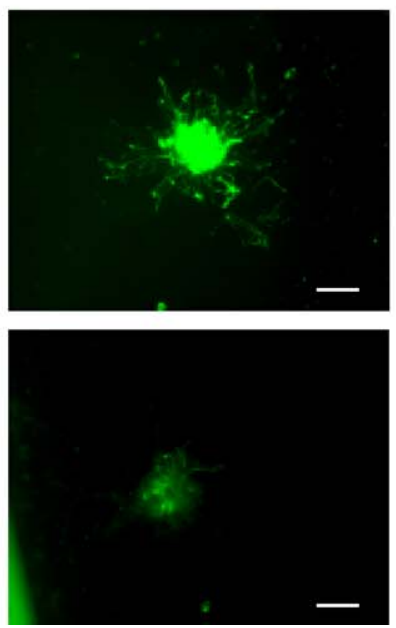

$\mathrm{O}_{4}$
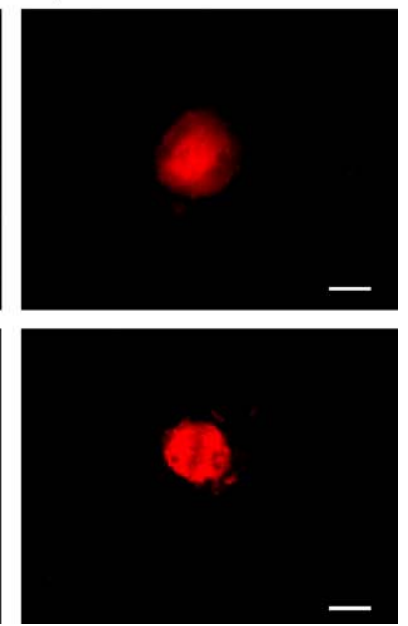

Fig. 4. Fluorescent photomicrographs represent differentiated cell phenotypes from embryonic cerebral cortical bFGF-responsive neurospheres on LAS and PDL substrates under serum-free conditions at low neurosphere density $\left(120\right.$ neurospheres $\left./ \mathrm{cm}^{2}\right)$ after $3 \mathrm{~d}$ of culture. Anti- $\beta$-tubulin-III and anti-MAP2 are immunoreactive for differentiated neurons, while anti-GFAP is specific for astrocytes, and anti-O4 is for oligodendrocytes. Scale bar $=100 \mu \mathrm{m}$.

against $\beta$-tubulin-III recognizes neurons at an early stage of development and is used to identify cells differentiating toward a neuronal phenotype $[25,29]$. Examination of the immunostaining results with $\beta$-tubulin-III-positive cells exhibited similar growth morphology on LAS and PDL substrates, suggested that the morphological growth of differentiating neurons is similar on these two substrates. On the other hand, the immunocytochemical distribution of MAP-2 is normally restricted to mature nerve cell bodies and dendrites [26,30]. Examination of the immunostaining results with MAP-2-positive cells revealed that the migrating behavior and morphological dendritic growth of these differentiated neurons were different on these two substrates. More mature neurons migrated away from neurospheres on PDL substrates and relatively fewer MAP-2-immuopositive cells were observed around the neurospheres on LAS substrates. In addition, most of the GFAP-positive cells, which had long and slender processes, show fibrous astrocyte morphology on LAS and PDL substrates [22]. However, O4-immunoreactive cells were not found on these two substrates. Based on the above results, neurosphere-forming cells differentiated predominantly into neurons and astrocytes. Therefore, these bFGF-responsive cerebral cortical neurospheres cultured on LAS or PDL substrates under this culture condition were bi-potent: they could differentiate into neurons and astrocytes simultaneously.

Fig. 5 shows the average percentage of differentiated cell phenotypes on LAS and PDL substrates, counted from MAP-2- and GFAP-positive cells of immunocytochemical staining in 20 random high-power fields of independent neurospheres from six independent cultures. After $3 \mathrm{~d}$ of differentiation in the serum-free medium, the percentages of differentiated neurons and astrocytes were $74.8 \pm 4.0 \%$ and $25.2 \pm 3.1 \%$ on PDL substrates, and were $52.2 \pm 5.9 \%$ and $47.9 \pm 4.3 \%$ on LAS substrates.

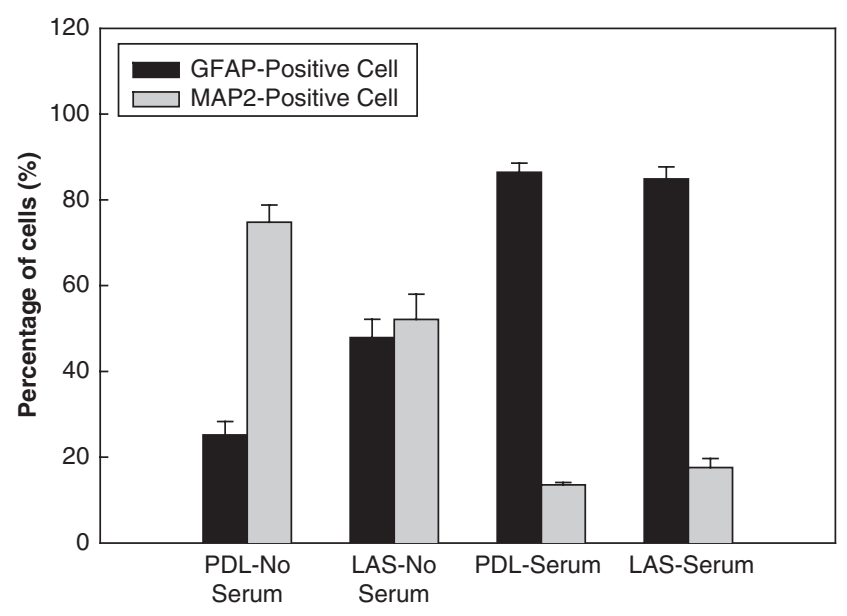

Fig. 5. Average percentage of differentiated cell phenotypes generated from neurospheres on LAS and PDL substrates in the presence or absence of serum medium.

\subsection{Behavior of neurospheres on LAS substrates at high density under serum-free conditions}

For the next experiment, the effect of neurosphere density on the behavior of cortical stem cells on LAS substrates was investigated. When the neurosphere density was increased to 360 neurospheres $/ \mathrm{cm}^{2}$ under serum-free conditions, neurospheres showed distinct behavior compared to that at low neurosphere density (120 neurospheres $/ \mathrm{cm}^{2}$ ). As shown in Fig. 6(a), extensive outgrowth of processes was not found after $3 \mathrm{~d}$ of culture. Conversely, neurosphere-forming cells migrated out from their original aggregate. It seemed that neurospheres broke down gradually. After migrating out of neurospheres, the cells became ovoid and bipolar or tripolar in shape with short processes. These cells were characterized as GFAP-positive 

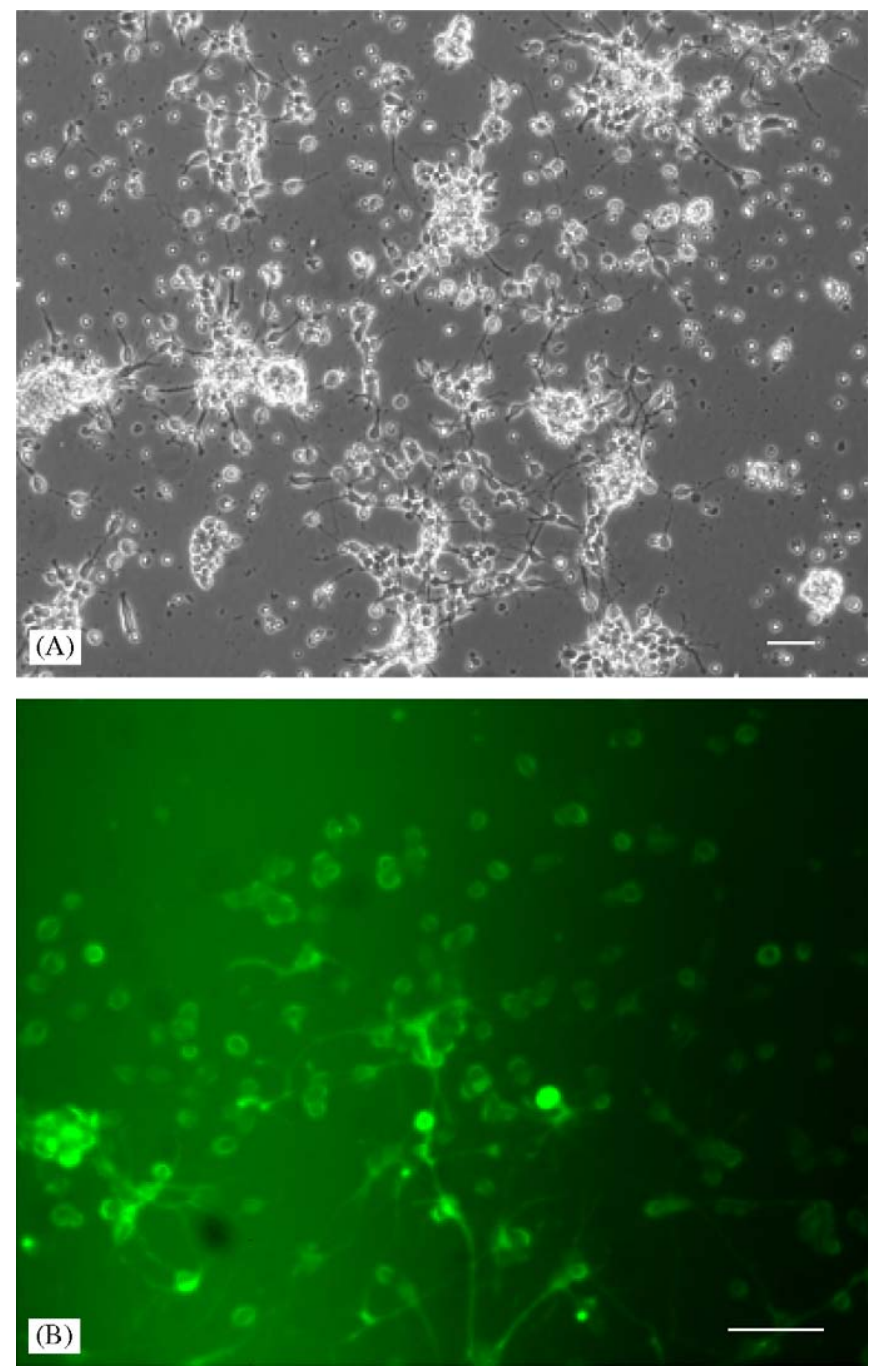

Fig. 6. (A) Morphology and (B) GFAP-immunoreactive cells of embryonic cerebral cortical neurospheres on LAS substrates under serum-free conditions at high neurosphere density (360 neurospheres/ $\mathrm{cm}^{2}$ ) after $3 \mathrm{~d}$ of culture. Scale bar $=100 \mu \mathrm{m}$.

cells (Fig. 6(b)), but MAP-2- and O4-negative (not shown here), indicating that migrating-out cells were predominantly induced into astrocytes. Therefore, increased neurosphere density can greatly influence the behavior and differentiated cell types of cortical neurospheres on LAS substrates.

\subsection{Behavior of neurospheres on LAS substrates at low density in the presence of serum}

Finally, we investigated the behavior of bFGF-responsive neurospheres on LAS substrates at low neurosphere density $\left(120\right.$ neurospheres $\left./ \mathrm{cm}^{2}\right)$ in the presence of $10 \%$ FBS. After $3 \mathrm{~d}$ of differentiation, the morphology of bFGF-responsive neurospheres grown on LAS substrates in the presence of serum was dramatically different from that grown in absence of serum. As shown in Fig. 7, the neurospheres were induced into typically an extensive
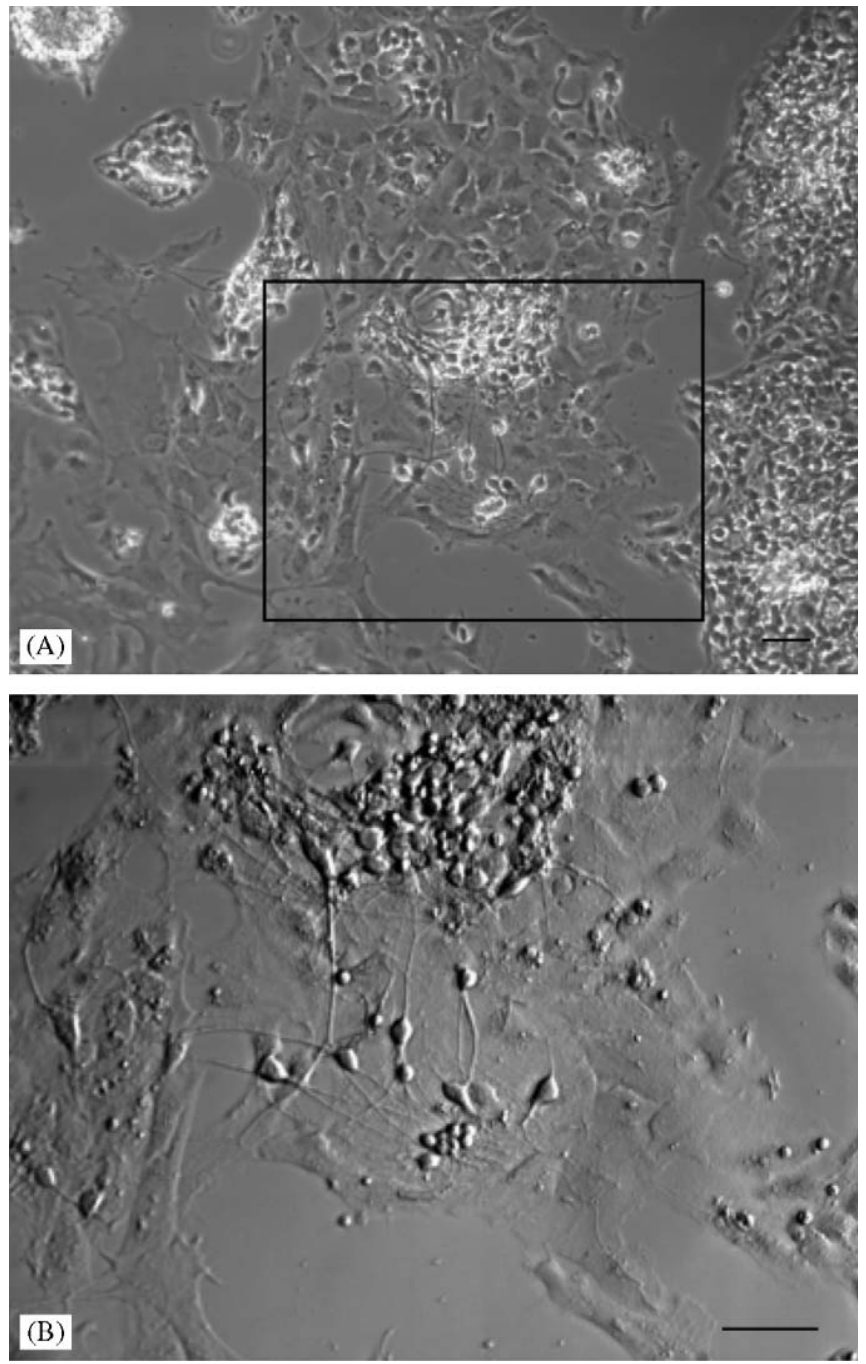

Fig. 7. (A) Morphology of embryonic cerebral cortical neurospheres on LAS substrates in the serum medium after $3 \mathrm{~d}$ of culture. (B) Enlarged views of boxed areas in (A), high-power DIC photomicrograph shows process-bearing cells grown on cytoplasmic spreading cells rather on LAS substrates. Scale bar $=100 \mu \mathrm{m}$.

cellular substratum of protoplasmic cells upon which process-bearing cells spread. The protoplasmic cells, exhibiting large, flat cell bodies with very few processes, would migrate far away from the neurospheres and were characterized as GFAP-immunoreactive astrocytes, and the process-bearing cells grown on these protoplasmic astrocyte layers were identified as either neurons (antiMAP-2, and anti $\beta$-tubulin-III) or astrocytes (anti-GFAP); see Fig. 8. This indicated that the processes grown on the substratum of astrocyte layers consist of MAP-2- and $\beta$-tubulin-III-positive neurofilaments from neurons and GFAP-positive processes from astrocytes. Therefore, when serum was present, the differentiated GFAP-positive astrocytes could be protoplasmic astrocytes or fibrous astrocytes. It is noted that protoplasmic astrocytes were not observed in the serum-free medium.

Fig. 5 also shows the average percentage of differentiated cell phenotypes on LAS and PDL substrates in the 

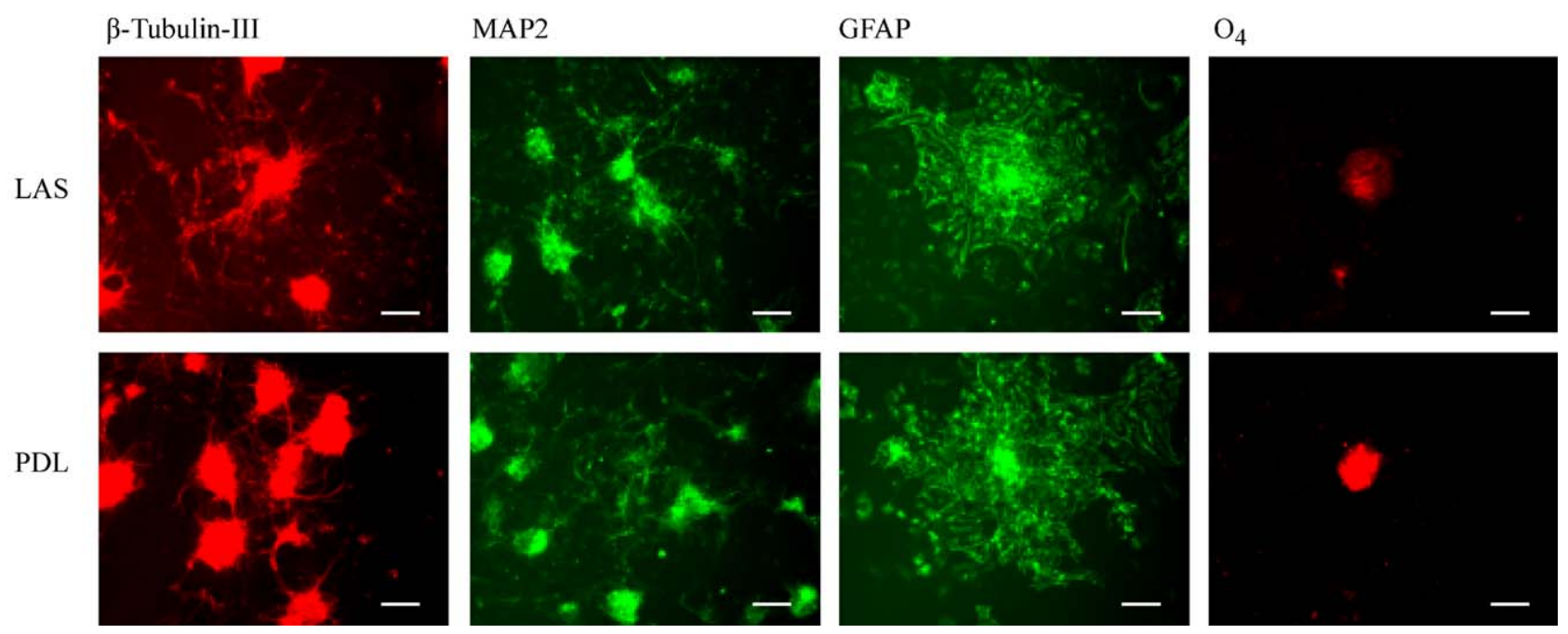

Fig. 8. Fluorescent photomicrographs represent differentiated cell phenotypes from embryonic cerebral cortical bFGF-responsive neurospheres on LAS and PDL substrates in the medium containing $10 \%$ FBS after $3 \mathrm{~d}$ of culture. Anti- $\beta$-tubulin-III and anti-MAP- 2 are immunoreactive for differentiated neurons, while anti-GFAP is specific for astrocytes, and anti-O4 is for oligodendrocytes. Scale bar $=100 \mu \mathrm{m}$.

presence of $10 \%$ FBS. When neurospheres were cultured on PDL substrates in the $10 \%$ FBS medium, the morphology and differentiated phenotypes of cortical neurospheres were similar as those observed on LAS substrates (Fig. 8). After $3 \mathrm{~d}$ of differentiation, the percentages of differentiated neurons and astrocytes were $13.6 \% \pm 0.5 \%$ and $86.4 \% \pm 2.1 \%$ on PDL substrates, and were $17.6 \% \pm 2.1 \%$ and $84.9 \% \pm 2.8 \%$ on LAS substrates. Clearly, regardless of LAS and PDL substrates, the percentage of differentiated neurons was decreased to a great extent when serum was present.

\subsection{MTT assay}

MTT assay relies on the ability of the viable cells to reduce a water-soluble yellow dye to a water-insoluble purple formazan product. Fig. 9 shows the MTT conversion of neurospheres cultured on the LAS substrates and TCPS at 120 neurospheres $/ \mathrm{cm}^{2}$. The effect of serum on the MTT reduction activity of cells was noted. At all data points, the MTT conversion of neurospheres in the presence of serum was greatly higher than that in the absence of serum, regardless on the LAS substrates and TCPS. In addition, the MTT reduction activity of cells on the LAS substrates in the presence of serum exhibited a stable increase and was significantly higher than that on TCPS at all data points $(p<0.001)$, indicating that the LAS substrate is favorable for the proliferation of neural stem cells in the presence of serum. This is consistent with the result of Fig. 5 that the percentage of differentiated astrocytes on LAS substrates was greatly increased when serum was present. However, there was no significant difference in the MTT conversion on LAS substrates and TCPS in the absence of serum. Therefore, the cell number would greatly increase on LAS substrates when only serum was present.

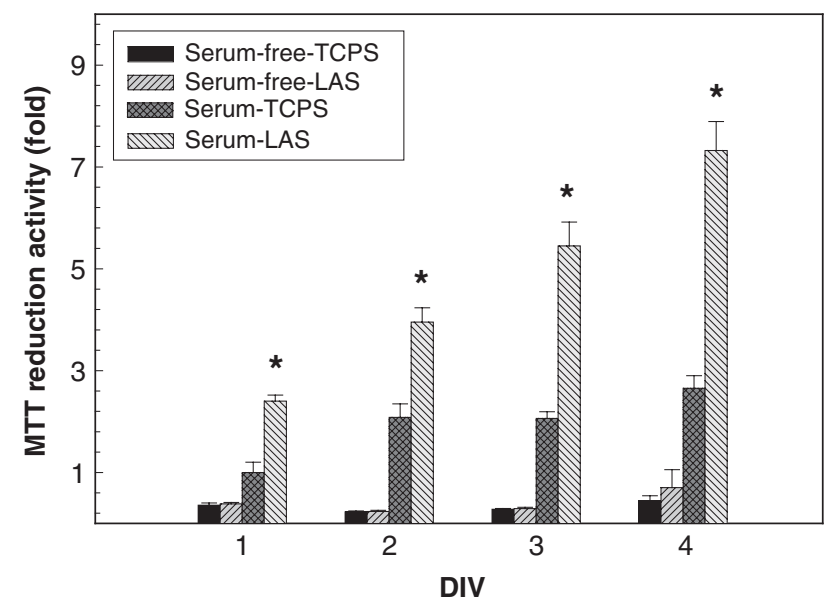

Fig. 9. MTT reduction activity of neurospheres cultured on LAS substrates and TCPS in the presence and absence of serum. The data were expressed as fold growth relative to seeding neurospheres \pm SEM from six independent cultures and determinations. Asterisk denotes significant differences of MTT reduction activity on LAS substrates compared to that on TCPS $(p<0.001)$ as determined by Student's $t$-test.

\section{Discussion}

Recently, the factors responsible for proliferation and differentiation of neural stem cells have been the subject of intense investigation because identification of effects that regulate the behavior of neural stem cells may allow for their eventual manipulation in the CNS therapies to replace cells lost to injury or disease. These factors include extrinsic signals coming from culture substrates [2,31], medium components $[3,5,7,22,32]$, and several complex interactions among cells $[6,10,33]$ to either augment or inhibit cellular proliferation or to impart new functional capabilities of neural stem cells. The major finding of this 
study was that neurospheres, obtained from embryonic rat cerebral cortex, could exhibit different behaviors on LAS according to the complex environment conditions.

Firstly, when neurospheres were sparsely distributed on LAS under serum-free conditions, long and straight processes extended from neurosphere to the surrounding areas. Albrecht-Buehler has reported that a mammalian cell can sense the presence of another cell at the range of cell diameter [34]. Therefore, it is reasonable to assume that neurospheres did not know whether other neurospheres existed around somewhere when the distance between neurospheres was so far that signals from soluble factors secreted by other neurospheres, such as hormones, cytokines and growth factors are extremely dilute [35]. At this time, if cells migrated far away from neurosphere, cells could not survive due to too low cell density [36,37]. Therefore, cells only could migrate a short distance around neurospheres. Alternatively, processes extended out from the neurosphere to search other neurospheres. It seemed that processes extending out from the neurosphere were a more economical method to communicate with other neurospheres than cells migrating out from the neurosphere. Therefore, processes extending from individual neurosphere could form a network between neurospheres, but only some migrating cells were found around the neurospheres. At this time, cell-substrate interaction was believed to determine pattern of process growth. As shown in Figs. 1-4, although the differentiated cell types (neurons and astrocytes) were similar on LAS and PDL substrates, process outgrowth was significantly enhanced by LAS substrates and morphological differentiation was somehow different on these two substrates.

If one places a neurosphere at the midpoint between two adjacent neurospheres, the long processes between two neurospheres will be intercepted by the third neurosphere (i.e., increasing neurosphere density). Theoretically, neurospheres should form a more tight and complex network under this circumstance because the distance between neurospheres is decreased substantially. However, when neurosphere density was increased from 120 to 360 neurospheres $/ \mathrm{cm}^{2}$ in this study, extensive outgrowth of processes was not found but astrocytes migrated out from their original aggregate to interact with other neurospheres (Fig. 6(b)). Indeed, the average number of migrating-out cells at the third day, counted from 15 neurospheres on six different substrates at low and high neurosphere density were $18.1 \pm 1.7$ and $45.5 \pm 3.9$, respectively. It seemed that neurospheres would change their social communication, which did not require physical contact, when they detected each other's presence. If the distance between neurospheres was near enough for cell migration to communicate with each other (i.e., higher neurosphere density), ovoid shaped astrocytes with short processes would migrate out from neurosphere to contact other cells. Conversely, if the distance was too far (i.e., lower density culture) for cells to migrate, longer processes extended outwards from cells inside the neurospheres instead of cell migration. This suggests that neurosphere density may considerably influence the behavior of cortical neurospheres.

Finally, when serum proteins were added to the culture system at low-density culture, extensive outgrowth of processes was not found but neurosphere-forming cells migrated out from their original aggregate. It appeared that serum proteins could alter communication between remote neurospheres. Suppose that the density-dependent increase in cell migration may be due to specific interactions between neurospheres controlled by growth factors (hormones or cytokines) secreted by cells [35]. Thus, a necessary condition for cells to migrate outside of the neurosphere is that signals from soluble growth factors secreted by other neurospheres needs to exceed a threshold. It can be inferred that the higher the neurosphere density, the shorter the mean distance between two adjacent neurospheres, and the higher the concentration of growth factor between neurospheres. This implies that the higher the neurosphere density, the easier for cells to migrate outside of the neurosphere. Thus, low levels of secreted growth factors by low density of neurospheres had no effect on the cell migration in sparse cultures. In contrast, sparse neurospheres could obtain strong signals when enough serum proteins are supplied to the medium. Therefore, the fate of neurospheres was changed from extensive outgrowth of processes to cells migrating out from their original aggregate. In other words, not only neurosphere density promotes cell migration but also the supply of serum proteins acts as a migration factor for cells in culture.

On the other hand, MTT assay shows the viability of neural stem cells in the presence of serum was significantly higher than that in the absence of serum (Fig. 9). As shown in Figs. 1 and 7, both in the same neurosphere density, serum proteins not only enhanced more cells to migrate out from neurospheres but also induced cells to differentiate into more different phenotypes; hence cells had higher mitochondria reactivity (MTT absorbance). Besides neurons, two types of astrocytes were induced and migrated out of neurospheres. One is the protoplasmic astrocyte with large, flat cell body with very few processes, and the other is the fibrous astrocyte with long processes and was grown on protoplasmic astrocyte layers. Such extensive cellular substratum of protoplasmic astrocytes differentiated from neurospheres can be considered as an optimum substrate for neuronal growth, which is similar to glia functioning as substrate pathways for the structuring of neuronal growth during CNS development [38-41]. On the contrary, when neurospheres were cultured under serumfree conditions, these neurosphere-forming cells could not migrate far away from neurospheres and survive within or around neurospheres. At this time, cells exhibited relatively low mitochondrial reactivity. It is noted that protoplasmic astrocytes were not observed under serum-free conditions. These suggested that serum proteins could exert much influence on growth and fate determination of neural stem cells. 


\subsection{Conclusion}

In summary, neurospheres would adopt different strategies to communicate with other neurospheres on LAS substrates according to the complex environmental conditions. Under serum-free conditions, neurosphere-forming cells could extend long processes to form a network between remote neurospheres or migrate out from their original aggregate to communicate with adjacent neurospheres. When serum was added to the culture system, serum proteins could alter communication between neurospheres. Even in sparse cultures, the fate of neurospheres was changed from extensive outgrowth of processes to cells migrating out from neurospheres. The present study provides evidence that LAS polymer can be a potent substrate for the development and growth of neural stem cells in tissue engineering and neuroscience research.

\section{Acknowledgement}

The authors thank National Taiwan University Hospital and National Science Council of the Republic of China for their financial support of this research.

\section{References}

[1] Mckay R. Stem cells in the central nervous system. Science 1997;276:66-70.

[2] Reynolds BA, Weiss S. Generation of neurons and astrocytes from isolated cells of the adult mammalian central nervous system. Science 1992;255:1707-10.

[3] Cattaneo E, McKay R. Proliferation and differentiation of neuronal stem cells regulated by nerve growth factor. Nature 1990;347:762-5.

[4] Davis AA, Temple S. A self-renewing multipotential stem cell in embryonic rat cerebral cortex. Nature 1994;372:263-5.

[5] Johe KK, Hazel TG, Muller T, Dugich-Djordjevic MM, McKay RD. Single factors direct the differentiation of stem cells from the fetal and adult central nervous system. Genes Dev 1996;10:3129-40.

[6] Tsai RY, McKay RD. Cell contact regulates fate choice by cortical stem cells. J Neurosci 2000;20:3725-35.

[7] Ciccolini F, Svendsen CN. Fibroblast growth factor 2 (FGF-2) promotes acquisition of epidermal growth factor (EGF) responsiveness in mouth striatal precursor cells: Identification of neural precursors responding to both EGF and FGF-2. J Neurosci 1998;18:7869-80.

[8] Arsenijevic Y, Weiss S, Schneider B, Aebischer P. Insulin-like growth factor-1 is necessary for neural stem cell proliferation and demonstrates distinct actions of epidermal growth factor and fibroblast growth factor-2. J Neurosci 2001;21:7194-202.

[9] Shimazaki T, Shingo T, Weiss S. The ciliary neurotrophic factor/ leukemia inhibitory factor/gp 130 receptor complex operates in the maintenance of mammalian forebrain neural stem cells. J Neurosci 2001;21:7642-53.

[10] Young TH, Hung CH. Behavior of embryonic rat cerebral cortical stem cells on the PVA and EVAL substrates. Biomaterials 2005;26:4291-9.

[11] Schmidt CE, Leach JB. Neural tissue engineering: strategies for repair and regeneration. Annu Rev Biomed Eng 1993;5:293-347.

[12] Geller HM, Fawcett JW. Building a bridge: engineering spinal cord repair. Exp Neurol 2002;174(2):125-36.

[13] Yavin E, Yavin Z. Attachment and culture of dissociated cells from rat embryo hemispheres on poly D-lysine coated surface. J Cell Biol 1974;62:540-6.

[14] Banker GA, Cowan WM. Rat hippocampal neurons in dispersed cell culture. Brain Res 1977;126:397-425.
[15] Mattson MP, Dou P, Kater SB. Outgrowth-regulating actions of glutamate in isolated hippocampal pyramidal neurons. J Neurosci 1988;8:2087-100.

[16] Brewer G, Cotman C. Survival and growth of hippocampal neurons in defined medium at low density: advantage of a sandwich culture technique or low oxygen. Brain Res 1989;494:65-74.

[17] Young TH, Hu WW. Covalent bonding of lysine to EVAL membrane surface to improve survival of cultured cerebellar granule neurons. Biomaterials 2003;24:1477-86.

[18] Brewer GJ, Deshmane S, Ponnusamy E. Precocious axons and improved survival of rat hippocampal neurons on lysine-alanine polymer substrates. J Neurosci Meth 1998;85:13-20.

[19] Bottenstein JE, Sato GH. Growth of a rat neuroblastoma cell line in serum-free supplemented medium. Proc Natl Acad Sci USA 1979;76:514-7.

[20] Engel J, Kurtz J, Katchalski KE, Berger A. Polymer tripeptides as collagen models. II. Conformational changes of poly (L-prolylglycylL-prolyl) in solution. J Mol Biol 1966;17:255-72.

[21] Wong G, Goldshmit Y, Turnley AM. Interferon- $\gamma$ but not TNF $\alpha$ promotes neuronal differentiation and neurite outgrowth of murine adult neural stem cells. Exp Neurol 2004;187:171-7.

[22] Chiang YH, Silani V, Zhou FC. Morphological differentiation of astroglial progenitor cells from EGF-responsive neurospheres in response to fetal calf serum, basic fibroblast growth factor, and retinal. Cell transplant 1996;5(2):179-89.

[23] Mosmann T. Rapid colorimetric assay for cellular growth and survival: application of proliferation and cytotoxicity assays. J Immunol Meth 1983;65:55-63.

[24] Deckwerth TL, Johnson Jr EM. Temporal analysis of events associated with programmed cell death (apoptosis) of sympathetic neurons deprived of nerve growth factor. J Cell Biol 1993;123:1207-22.

[25] Dráberová E, Lukás Z, Ivanyi D, Viklický V, Dráber P. Expression of class III beta-tubulin in normal and neoplastic human tissue. Histochem Cell Biol 1998;109:231-9.

[26] Weclewicz K, Svensson L, Billger M, Holmberg K, Wallin M, Kristensson K. Microtubule-associated protein 2 appears in axons of cultured dorsal root ganglia and spinal cord neurons after rotavirus infection. J Neurosci Res 1993;36:173-82.

[27] Bignami A, Eng LF, Dahl D, Uyeda CT. Localization of the glial fibrillary acidic protein in astrocytes by immunofluorescence. Brain Res 1972;43:429-35.

[28] Sommer I, Schachner M. Monoclonal antibody (O1 to O4) to oligodendrocyte cell surfaces: an immunocytological study in the central nervous system. Dev Biol 1981;83:311-27.

[29] Duittoz AH, Hevor T. Primary culture of neural precursors from the ovine central nervous system (CNS). J Neurosci Meth 2001;107:131-40.

[30] Diez-Guerra FJ, Avila J. An increase in phosphorylation of microtubule associated protein 2 accompanies dendrite extension during the differentiation of cultured hippocampal neurons. Eur J Biochem 1995;227:68-77.

[31] Shetty AK, Turner DA. In vitro survival and differentiation of neurons derived from epidermal growth factor-responsive postnatal hippocampal stem cells: inducing effects of brain-derived neurotrophic factor. J Neurobiol 1998;35:395-425.

[32] Reynolds BA, Tetzlaff W, Weiss S. A multipotent EGF-responsive striatal embryonic progenitor cells produces neurons and astrocytes. J Neurosci 1992;12:4565-74.

[33] Barakat I, Sensenbrenner M, Vincendon G. The importance of cell contact for the proliferation of neuroblasts in culture and its stimulation by meningeal extract. Neurochem Res 1982;7:287-300.

[34] Albrecht-Buehler G. A long-range attraction between aggregating 3 T3 cells mediated by bear-infrared light scattering. Proc Natl Acad Sci USA 2005;102:5050-5.

[35] Young TH, Huang JH, Hung SH, Hsu JP. The role of cell density in the survival of cultured cerebellar granule neurons. J Biomed Mater Res 2000;52:748-53. 
[36] Kilpatrick TJ, Bartlett PF. Cloning and growth of multipotential neural precursors: requirements for proliferation and differentiation. Neuron 1993;10:255-65.

[37] Hulspas R, Tiarks C, Reilly J, Hsieh CC, Recht L, Quesenberry PJ. In vitro cell density-dependent clonal growth of EGF-responsive murine neural progenitor cells under serum-free conditions. Exp Neurol 1997;148:147-56.

[38] Noble M, Fok-Seang J, Cohen J. Glia are a unique substrate for the in vitro growth of central nervous system neurons. J Neurosci 1984;4(7):1892-903.
[39] Varon S. Neurons and glial in neuronal cultures. Exp Neurol 1975;48:93-134.

[40] Gasser UE, Hatten ME. Neuron-glia interactions of rat hippocampal cells in vitro: glial guided neuronal migration and neuronal regulation of glial differentiation. J Neurosci 1990;10(4):1276-85.

[41] Costa S, Planchenault T, Charriere-Bertrand C, Mouchel Y, Fages C, Juliano $\mathrm{S}$, et al. Astroglial permissivity for neuritic outgrowth in neuron-astrocyte coculture depends on regulation of laminin bioavailability. Glia 2002;37:105-13. 